\section{Design of a Wideband Antenna with Stable Omnidirectional Radiation Pattern Using the Theory of Characteristic Modes}

\author{
Dingliang Wen, Yang Hao, Hanyang Wang and Hai Zhou
}

\begin{abstract}
In this communication, the design procedure of a wideband antenna with omnidirectional radiation pattern is demonstrated based on the theory of characteristic modes. Consisting of a dipole and a loop antenna, the antenna has a very simple structure. A wide impedance bandwidth is obtained because of the simultaneous excitation of the antenna's first two modes. Meanwhile, due to the fact that these two modes share a similar omnidirectional radiation pattern, a stable radiation pattern is also achieved across the operating frequency band. In order to identify the antenna's different modes, a characteristic mode analysis of the antenna is carried out first. Then, a feed configuration is specifically designed to excite the desired modes. To validate the antenna design, a prototype was fabricated and tested. Measured results agree well with the simulated ones. Measurement shows that a wide impedance bandwidth of $44.2 \%$ with $\left|S_{11}\right|<-10 \mathrm{~dB}(1.85-2.9 \mathrm{GHz})$ and stable radiation patterns at both $\mathrm{E}$-plane and $\mathrm{H}$-plane were achieved over the operating frequency band.
\end{abstract}

Index Terms - Characteristic modes, multimode antenna, wideband antenna, omnidirectional antenna, stable radiation pattern.

\section{INTRODUCTION}

Recent communication services, such as $2 \mathrm{G}, 3 \mathrm{G}$, LTE, $4 \mathrm{G}$, Wi-Fi, and WiMax, boost the high demand of wideband antennas with wide impedance bandwidth and stable radiation patterns over their operating frequency bands. One approach to obtain a wide impedance bandwidth is by adopting frequency independent antenna concept [1], such as log-periodic antennas [2], spiral antennas [3], and conical antennas [4]. Unfortunately, these antennas [2-4] are always too complex and bulky in their geometrical structures, which makes them difficult to be fabricated. On the other hand, patch antennas have been widely studied for their simple structure and large numbers of feeding techniques were proposed to broaden their impedance bandwidths, such as slot coupling feeding [5], U-shaped slot feeding [6], L-shaped probe feeding [7], etc. Slot antenna is another good candidate for modern wireless communications for its simple and planar structure. A great number of papers about wideband slot antennas have been published recently [8-10]. An interesting paper about slot antenna was published in 2015 [11], in which a slot stub is added to shift the higher mode down to the lower mode and as a

Manuscript received February \#\#, 2016. This work was supported by Huawei Technologies (UK) CO., LTD.

D. L. Wen and Y. Hao are with the School of Electronic Engineering and Computer Science, Queen Mary College, University of London, London E1 4NS, U.K. (e-mail: dingliang.wen@qmul.ac.uk, y.hao@qmul.ac.uk).

H. Y. Wang and H. Zhou are with Huawei Technologies (UK) CO., LTD, Reading, Berkshire, U.K. (email: hanyang.wang@huawei.com, hai.zhou1@huawei.com). result, a wide impedance matching is achieved. However, all of these antennas [5-11] have a weakness of unstable radiation patterns across their operating frequency bandwidths.

Another approach to design wideband antenna is by using the theory of characteristic modes (TCM) [12-16]. The biggest advantage of it over other approaches is that the TCM gives us a direct insight into the radiating phenomena occurring on the antenna. Therefore, desired radiation patterns can be obtained by exciting the corresponding modes. Unfortunately, none of these authors [12-16] have considered obtaining stable radiation patterns while broadening the antennas' impedance bandwidth by utilizing the TCM.

To obtain a stable radiation pattern, the concept of magneto-electric dipole antenna was first proposed [17] in 2006. By combining an electric planar dipole and a shorted patch, which is treated as a magnetic dipole, a stable radiation pattern is achieved over its operating frequencies. Another way to achieve a stable radiation pattern is by using multi-dipole antenna, the principle of which is explained in detail in [18]. By using two pairs of dipoles [18], a wide impedance bandwidth of $60 \%$ with $\mathrm{SWR}<1.5$ and a stable radiation pattern across the operating frequency band are obtained. Other antenna designs with stable radiation patterns have also been investigated recently for base station systems [19-21].

Sometimes, an omnidirectional radiation pattern is more preferable in wireless communications while all of the antennas' radiation patterns in [17-21] are unidirectional. In this communication, a wideband antenna with stable omnidirectional radiation pattern is proposed according to the theory of characteristic modes. The TCM gives us a direct insight into the radiating phenomenon taking place on the proposed antenna and explains how stable radiation patterns are obtained over the whole operating frequency band. The proposed antenna can achieve a wide impedance bandwidth of $44.2 \% \quad(\mathrm{SWR}<2)$ from 1.85 to $2.9 \mathrm{GHz}$ and a stable omnidirectional radiation pattern at H-plane over its operating frequency band. The antenna also has a simple structure and is easy to be built by using PCB fabrication technique.

\section{Characteristic Modes’ Analysis}

\section{A. Theory of Characteristic Modes}

The theory of characteristic modes was first proposed by Garbacz and Turpin in 1971 [22], and then it was refined by Harrington and Mautz in 1970s [23, 24]. The TCM is a powerful tool to design antennas because it can provide a direct insight into the radiating phenomena occurring on the antenna. In recent years, it has been widely used to design all types of antennas, such as mobile handset antenna, electrically small antenna, platform antenna, planar monopole antenna, etc [25]. In this section, only basic concept of the TCM will be presented. The full definition and explanation of the TCM can be found in [22-24].

For a given conducting object, any electric current $(\vec{J})$ on its surface can be expressed as a sum of characteristic modes or eigencurrents $\left(\vec{J}_{n}\right)$ with different product coefficients $\left(\alpha_{n}\right)$ : 


$$
\vec{J}=\sum_{n} \alpha_{n} \vec{J}_{n}=\sum_{n} \frac{V_{n}^{i} \vec{J}_{n}}{1+j \lambda_{n}}
$$

where $\vec{J}_{n}$ are the eigencurrents, $\alpha_{n}$ are the modal weighting coefficients, $\lambda_{n}$ are the eigenvalues, and $V_{n}^{i}$ are the modal excitation coefficients, which is defined by:

$$
V_{n}^{i}=\left\langle\vec{J}_{n}, \overrightarrow{\mathrm{E}}^{i}\right\rangle=\oiint_{S} \vec{J}_{n} \cdot \overrightarrow{\mathrm{E}}^{i} d s
$$

where $\vec{E}^{i}$ is the impressed source. Modal weighting coefficient $\alpha_{n}$ can be expressed as follows:

$$
\alpha_{n}=\frac{V_{n}^{i}}{1+j \lambda_{n}}
$$

Modal significance $\left(M S_{n}\right)$ is always used to determine how efficiently a mode can radiate. The expression of $M S_{n}$ can be presented as:

$$
M S_{n}=\left|\frac{1}{1+j \lambda_{n}}\right|
$$

Another important parameter that will be utilized in the antenna design procedure is characteristic angle $\beta_{n}$, which is defined as:

$$
\beta_{n}=180^{\circ}-\tan ^{-1}\left(\lambda_{n}\right)
$$

When a mode $\vec{J}_{n}$ is resonant namely $\lambda_{n}=0, M S_{n}=1$ and $\beta_{n}=180^{\circ}$ can be obtained according to (2-4) and (2-5), respectively. Therefore, the mode radiates all of the energy when $\beta_{n}=180^{\circ}$ and the closer $\beta_{n}$ is to $180^{\circ}$ or $\lambda_{n}$ is to 0 , the more efficiently the mode radiates when excited. According to equation (2-1), how well a mode $\vec{J}_{n}$ is excited depends on the modal weighting coefficient $\alpha_{n}$. Meanwhile, $\alpha_{n}$ is dependent on two parameters, i.e., eigenvalue $\lambda_{n}$ and modal excitation coefficients $V_{n}^{i}$. For a resonant mode at its resonance frequency, $\lambda_{n}$ is equal to 0 so formula (2-3) can be changed to:

$$
\alpha_{n}=V_{n}^{i}
$$

However, for some non-resonant modes, their eigenvalues are very huge over the observed frequency band. This means that no matter how you excite the conducting object, which is antenna in our case, the modal weighting coefficient is always very small. We address these modes as insignificant modes. In our following study, only significant modes are taken into consideration, whose characteristic angles are close to $180^{\circ}$.

Based on formula (2-3), when eigenvalue $\lambda_{n}$ is fixed, the modal weighting coefficient is totally determined by how we excite the antenna. Then according to (2-2), in order to obtain a large value of modal weighting coefficient, the excitation should be placed where the eigencurrent is very strong.

\section{B. Characteristic Modes' Analysis}

In this section, a detailed analysis of characteristic modes of the proposed antenna will be given. Note that all of the simulations performed in this section was carried out by using CADFEKO Suite 7.0 [26]. To simplify the process, the

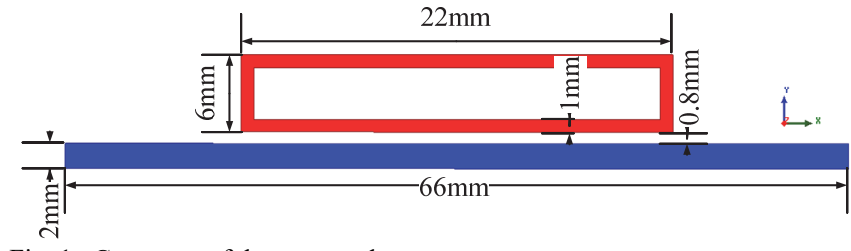

Fig. 1. Geometry of the proposed antenna.

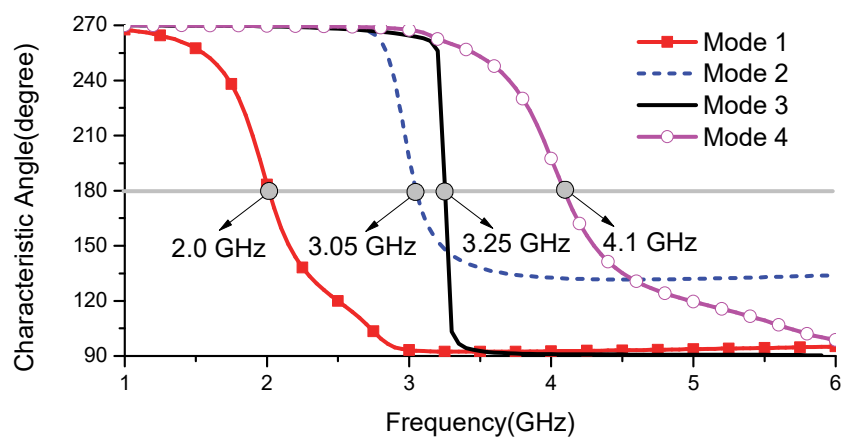

Fig. 2. Characteristic angles of the first four modes of the proposed antenna.

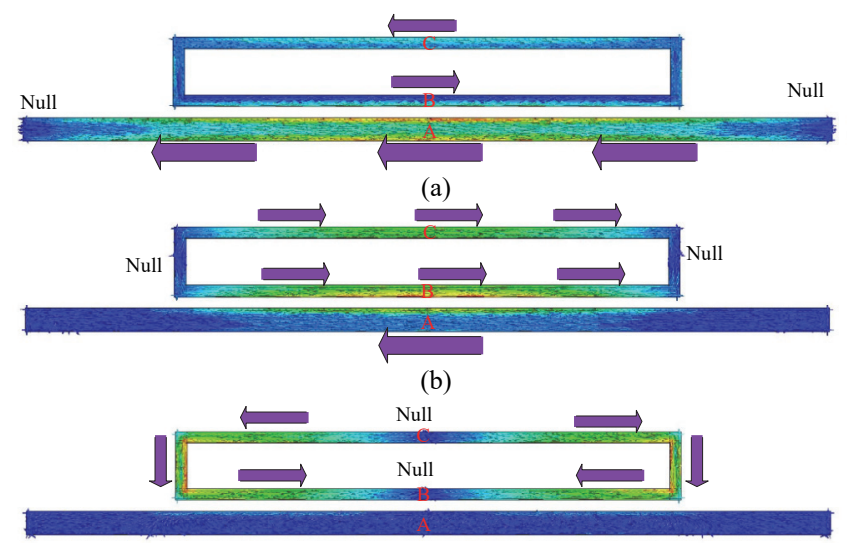

(c)

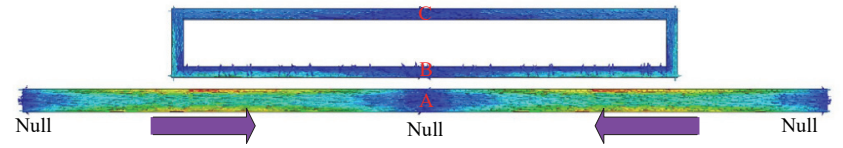

(d)

Fig. 3. Current distributions of (a) mode 1 at $2.0 \mathrm{GHz}$, (b) mode 2 at $3.05 \mathrm{GHz}$, (c) mode 3 at $3.25 \mathrm{GHz}$, and (d) mode 4 at $4.1 \mathrm{GHz}$ of the proposed antenna.

antenna to be analyzed in this section is set to be perfect conductor with zero thickness and without any substrates.

The idea of the proposed antenna was inspired by the fact that the dipole's half-wave mode and the folded dipole's one-wave mode share the similar omnidirectional radiation pattern. Basically, the antenna is a combination of a dipole and a loop antenna, as illustrated in Fig. 1. The proposed antenna's first four resonant modes characteristic angles are presented in Fig. 2. Fig. 3 and Fig. 4 illustrate these four modes' current distributions and radiation patterns at their resonant frequencies, respectively. The proposed antenna's first two modes are the modes we need because they share a similar omnidirectional radiation pattern, which can be observed in Fig. 4. Actually, it can be seen from Fig. 3 that these two modes are similar to dipole's half-wave mode and the folded dipole's one-wave mode, respectively. 


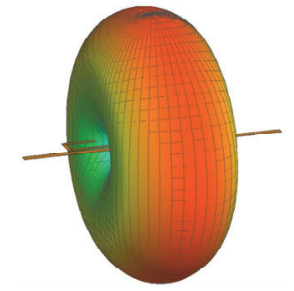

(a)

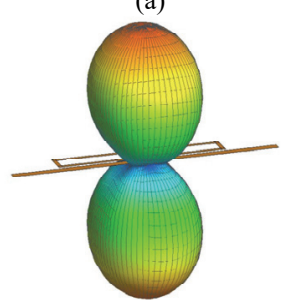

(c)

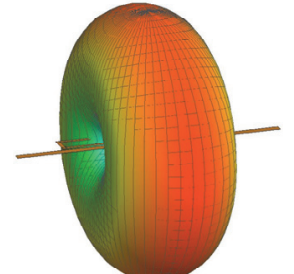

(b)

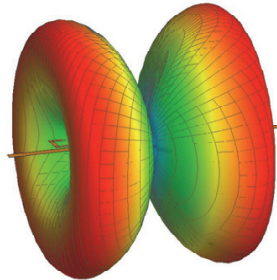

(d)
Fig. 4. Radiation patterns of (a) mode 1 at $2.0 \mathrm{GHz}$, (b) mode 2 at $3.05 \mathrm{GHz}$, (c) mode 3 at $3.25 \mathrm{GHz}$, and (d) mode 4 at $4.1 \mathrm{GHz}$ of the proposed antenna.

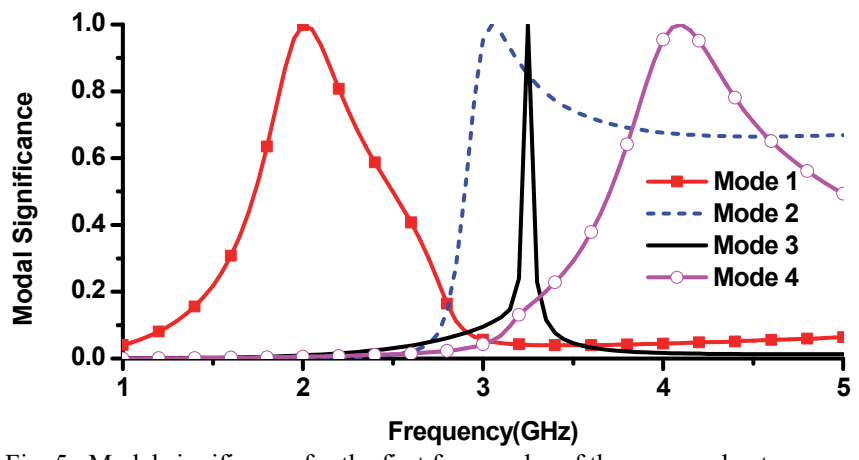

Fig. 5. Modal significance for the first four modes of the proposed antenna.

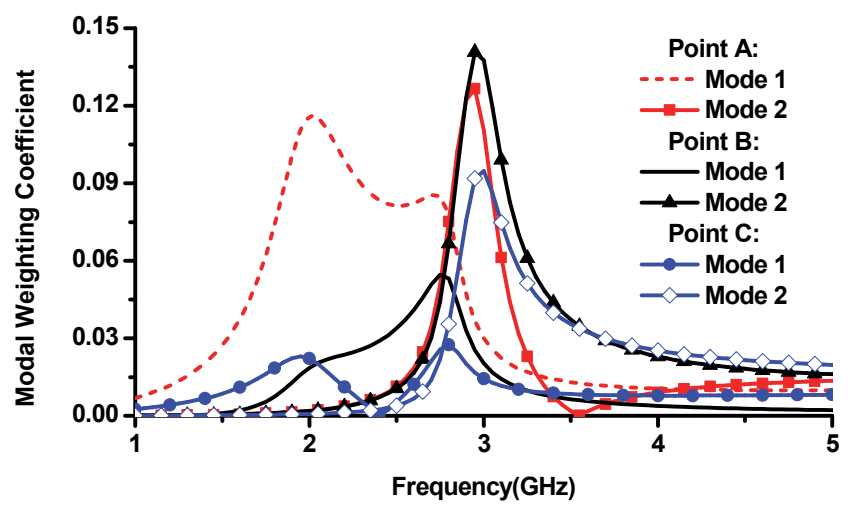

Fig. 6. Modal weighting coefficients for the first two modes of the proposed antenna with different feeding locations.

The next stage of the antenna design is to create a feeding structure to excite the proposed antenna's first two modes simultaneously. According to formula (2-2), to excite a certain mode, a direct feed should be located where the eigencurrent is very strong. Fig. 3(a) shows that the best feeding location for mode 1 is the middle of the dipole, namely point $\mathrm{A}$, where the current is the maximum while point $\mathrm{B}$ or $\mathrm{C}$ may be a second option. For mode 2, the best feeding place is point $\mathrm{B}$ or $\mathrm{C}$ while point $\mathrm{A}$ is also possible. In order to obtain the best feeding location, three cases will be discussed, i.e., point A, point B and point $C$. Modal significance for the first four modes are presented in Fig. 5. It can be seen that all of the modes have a

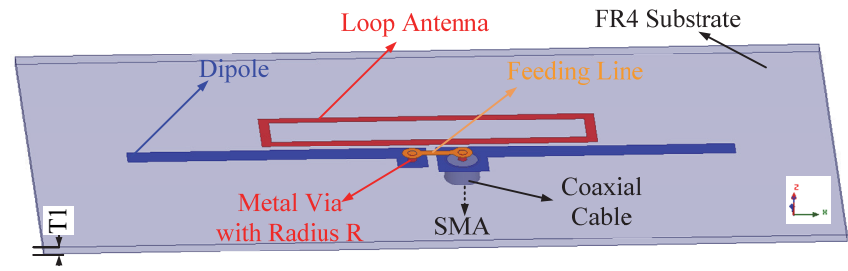

(a)

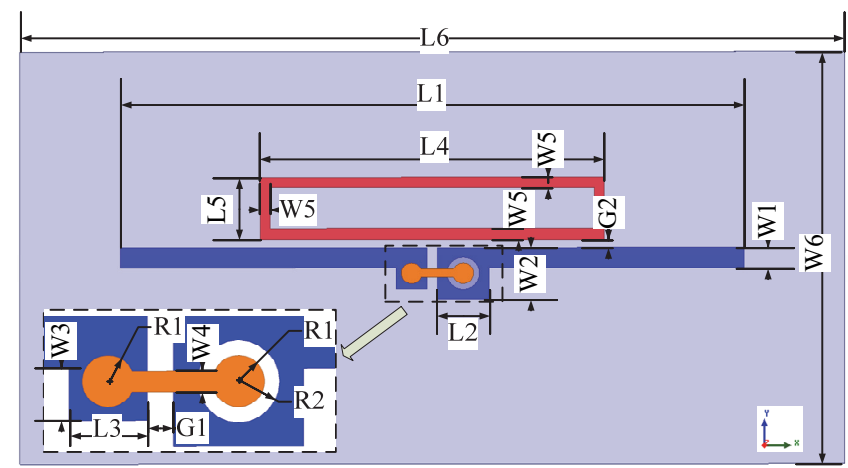

(b)

Fig. 7. Geometry of the proposed antenna: (a) 3-D view; (b) top view; $L 1=61.8$, $\mathrm{L} 2=5, \mathrm{~L} 3=3, \mathrm{~L} 4=32.2, \mathrm{~L} 5=6, \mathrm{~L} 6=80, \mathrm{~W} 1=2, \mathrm{~W} 2=\mathrm{L} 2, \mathrm{~W} 3=2, \mathrm{~W} 4=0.8, \mathrm{~W} 5=1$, $\mathrm{W} 6=40, \mathrm{R}=0.5, \mathrm{R} 1=1, \mathrm{R} 2=1.58, \mathrm{G} 1=1, \mathrm{G} 2=0.8, \mathrm{~T} 1=0.8$ (Units: $\mathrm{mm}$ ).

large modal significance around 1 at their resonant frequencies, which means the corresponding modes can radiate very efficiently around their resonant frequencies. However, neither mode 3 nor mode 4 can be excited in those previously described cases because their currents at points $\mathrm{A}, \mathrm{B}$ and $\mathrm{C}$ are nulls, as shown in Figs. 3(c) and (d). In other words, their modal weighting coefficients are very small though a large modal significance is observed at their resonant frequencies. Therefore, in the following study these two modes will not be considered.

Fig. 6 shows the modal weighting coefficients for the first two modes of the proposed antenna with different feeding locations, i.e., point $\mathrm{A}$, point $\mathrm{B}$ and point $\mathrm{C}$. As can be seen, when a voltage source is added at point $\mathrm{A}$, both mode 1 and mode 2 are strongly excited, especially around their resonant frequencies; in the other two cases, mode 2 is well excited but mode 1 is not excited equivalently even at its resonant frequency. Therefore, we can draw a conclusion that point $\mathrm{A}$ is the best feeding location.

\section{ANTENNA DESIGn ANd PARAMETRIC StUdy}

The geometry of the proposed antenna with detailed dimensions is shown in Fig. 7. It is designed using an $80 \mathrm{~mm} \times$ $40 \mathrm{~mm} \times 0.8 \mathrm{~mm} \mathrm{FR} 4$ substrate with a permittivity of 4.4 and a loss tangent of 0.02. As illustrated in Fig. 7(a), the antenna consists of a dipole, a loop antenna, a feeding line and a coaxial feed. Both the dipole and the loop antenna are printed on the lower side of the substrate while the feeding line is on the upper side.

A coaxial feed is selected to excite the proposed antenna. It has already been known from Section II that the optimum feeding location is the middle of the dipole. To implement this feed method, the dipole has been altered, as shown in Fig. 


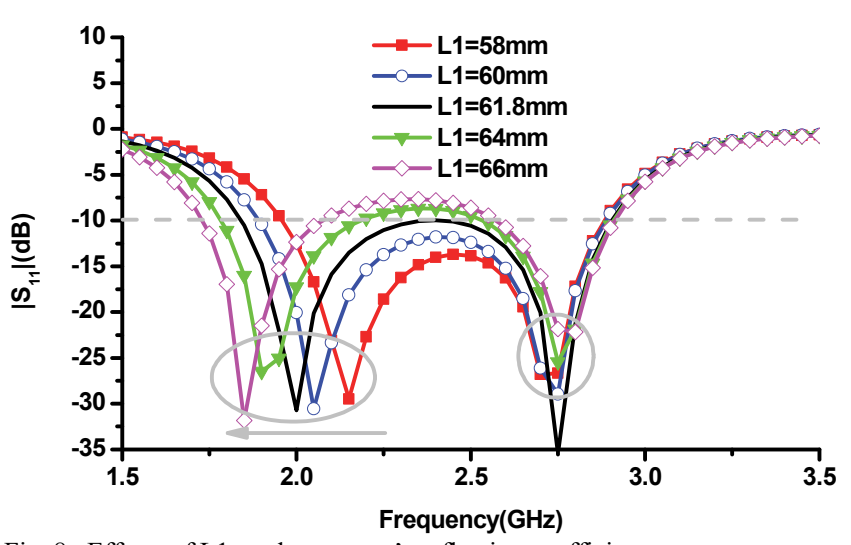

Fig. 8. Effects of $\mathrm{L} 1$ on the antenna's reflection coefficient.

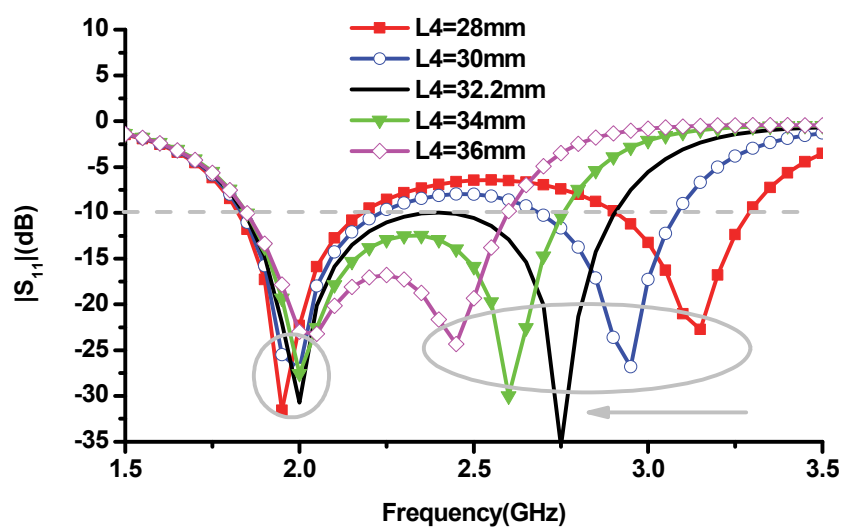

Fig. 9. Effects of L4 on the antenna's reflection coefficient.

7(b): a slit is made in the middle of it; the width of the dipole around the feeding structure is increased; an etched part is also made in the dipole's right arm. Fig. 7(a) shows that the outer conductor of the coaxial cable is soldered to the right arm of the dipole while the inner part is connected with the feeding line through the substrate. The other end of the feeding line is bonded to the left arm of the dipole using a metal via. Two holes with a diameter of $1 \mathrm{~mm}$ are drilled in the FR4 substrate to realize the feed.

To demonstrate the analyses presented in Section II, the effects of some key parameters of the proposed antenna on its reflection coefficient will be discussed in the following part, such as the length of the dipole L1, the loop antenna's side length L4 and L5, and the gap width between the dipole and the loop G2. Note that when one parameter is studied, the others are kept as a constant. All of the simulations in this section were carried out by using Ansoft HFSS v. 15 [27].

The first parameter studied was the length of the dipole L1. As seen in Fig. 8, the proposed antenna has two resonances from 1.5 to $3.5 \mathrm{GHz}$. When L1 equals $61.8 \mathrm{~mm}$, they occur at about $2.0 \mathrm{GHz}$ and $2.75 \mathrm{GHz}$, respectively. These two resonances correspond with the first two characteristic modes of the antenna, as discussed in Section II. Fig. 8 also shows that when L1 increases the first mode's resonant frequency shifts to a lower frequency; however, the second mode is insensitive to the change of L1. The reason for this phenomenon is that the first mode is caused by the dipole and the second mode is introduced by the loop antenna. Therefore, when the dipole's

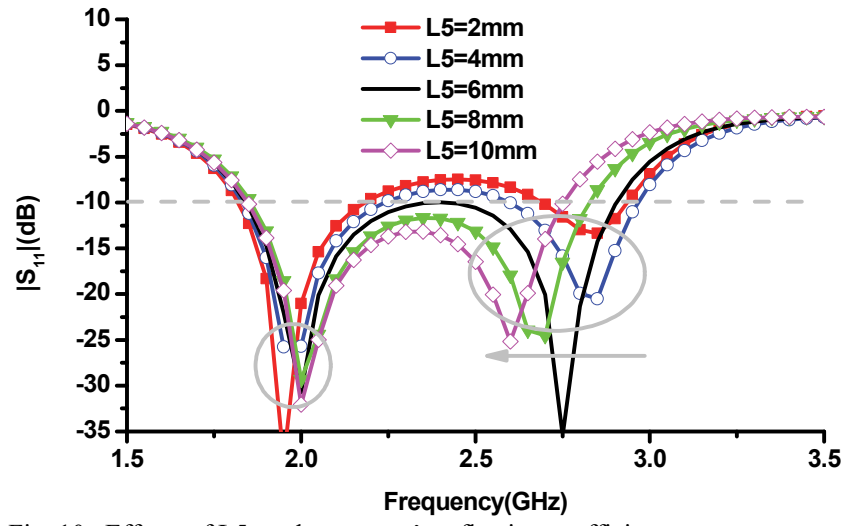

Fig. 10. Effects of L5 on the antenna's reflection coefficient.

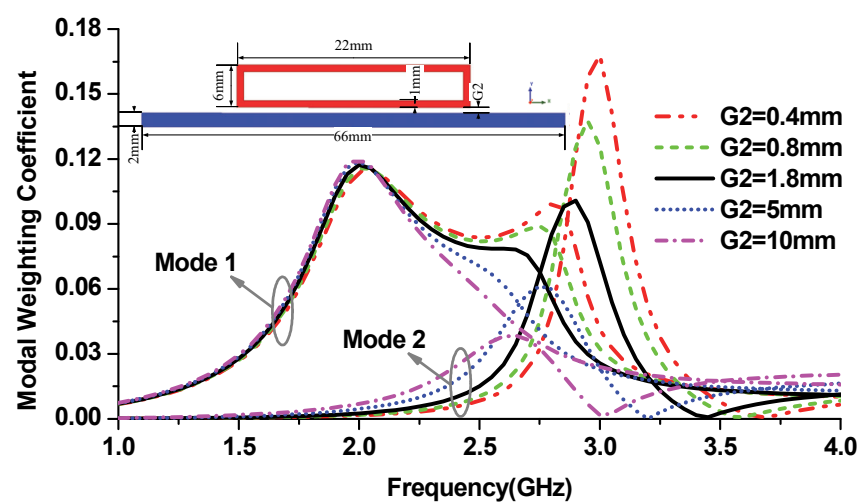

Fig. 11. Effects of G2 on the antenna's modal weighting coefficient.

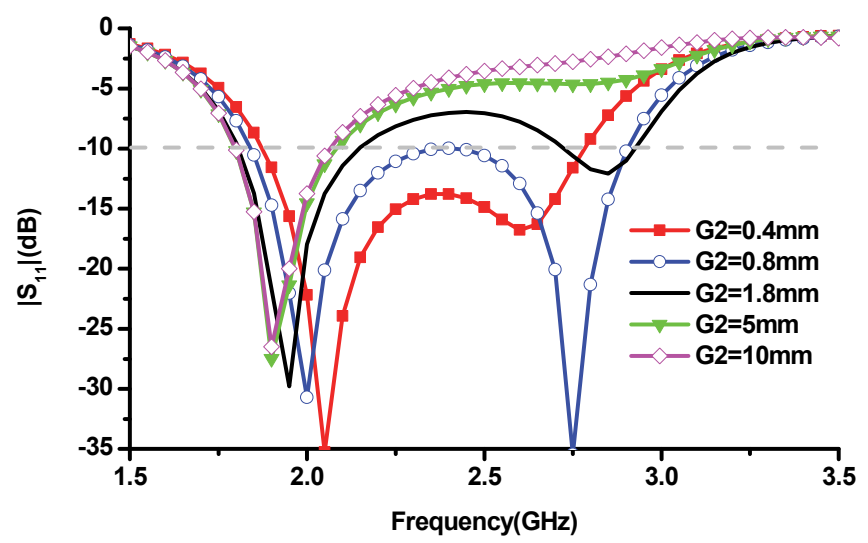

Fig. 12. Effects of G2 on the antenna's reflection coefficient.

length increases its resonance should occur at a lower frequency. In order to make the first mode resonates at a proper frequency, L1 was chosen to be $61.8 \mathrm{~mm}$.

The second and third parameters studied were the loop antennas' two sides' length, L4 and L5. Their effects on the proposed antenna's reflection coefficient are shown in Fig. 9 and 10 , respectively. It can be seen that the first mode's resonant frequency keeps unchanged when L4 or L5 increases while the second mode's resonance occurs at a lower frequency in both of the cases. These phenomena demonstrate that the first mode of the antenna is determined by the dipole while the second mode is caused by the loop antenna. Hence, for a wide impedance bandwidth, L4 and L5 were set to be $32.2 \mathrm{~mm}$ and 6 $\mathrm{mm}$, respectively. 


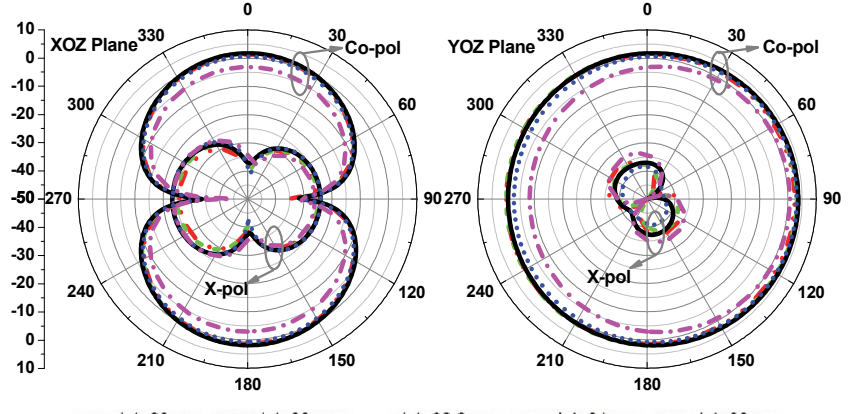

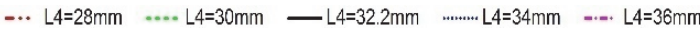
Fig. 13. Effects of L4 on the antenna's radiation patterns at $2.8 \mathrm{GHz}$.

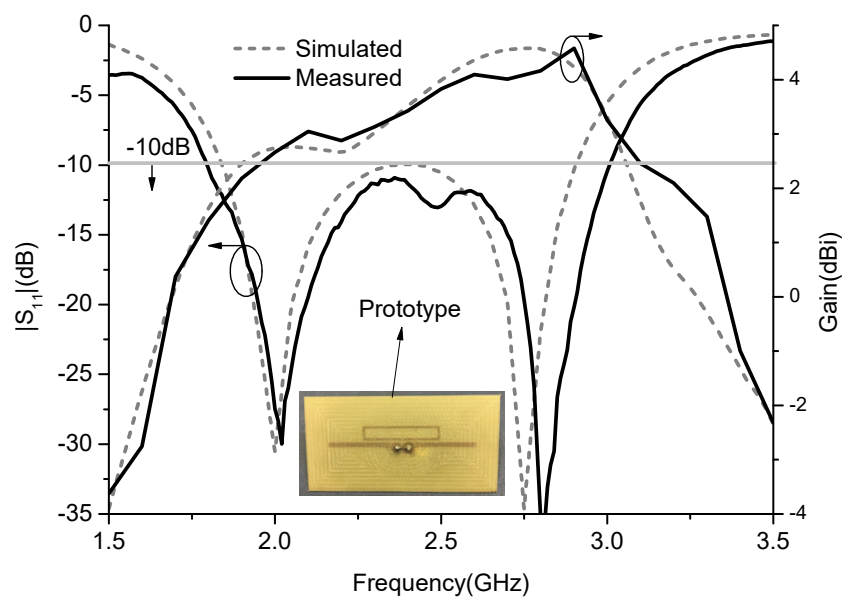

Fig. 14. Simulated and measured reflection coefficient and gain of the proposed antenna.

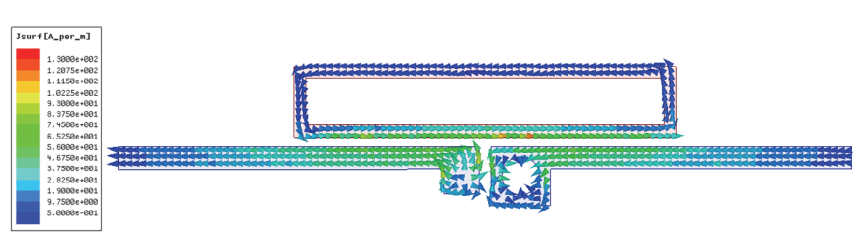

(a)

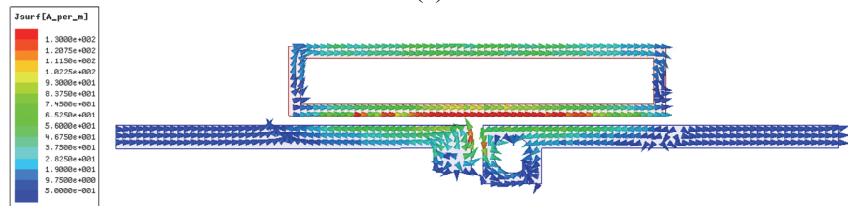

(b)

Fig. 15. Current distributions of the proposed antenna at (a) $2.0 \mathrm{GHz}$ (first resonance), (b) $2.75 \mathrm{GHz}$ (second resonance).

The fourth parameter studied was the gap width between the dipole and the loop G2. For the second resonance, the dipole works as a couple feeding structure of the loop antenna, so the gap width G2 determines how well the second mode can be excited. Fig. 11 shows that a greater value of G2 yields a smaller modal weighting coefficient of the second mode, namely a weaker excitation of this mode. It can be seen from Fig. 12 that when G2 increases from $0.4 \mathrm{~mm}$ to $10 \mathrm{~mm}$, the first resonance shifts slightly to a lower frequency while the second one moves gradually to a higher frequency. Additionally, as G2 is greater than $5 \mathrm{~mm}$ only the first mode can be excited. To achieve a good impedance matching over a wide frequency band, $\mathrm{G} 2=0.8 \mathrm{~mm}$ was selected.

These four parameters' (L1, L4, L5 and G2) impact on the

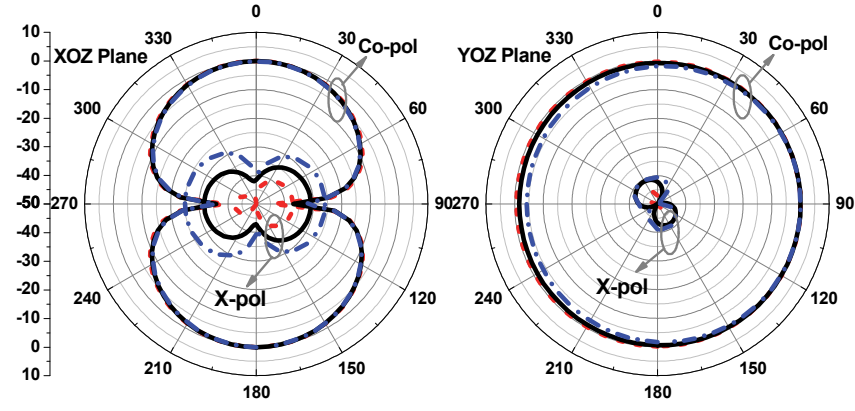

(a)

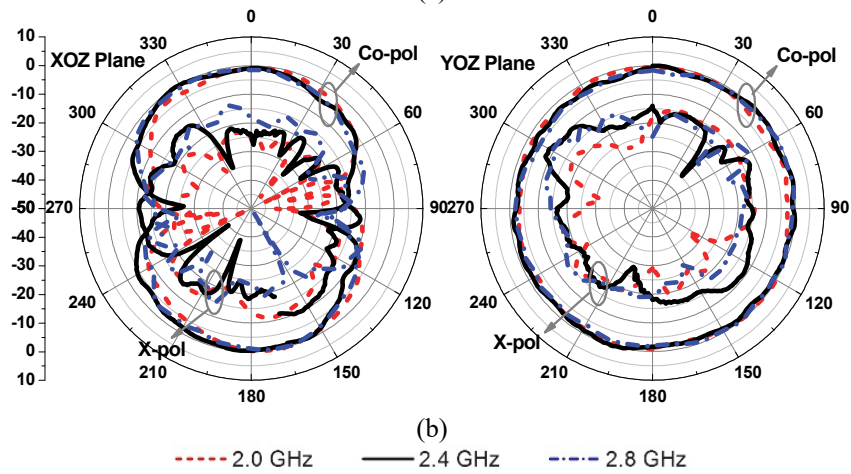

Fig. 16. (a) Simulated and (b) measured radiation patterns of the proposed antenna.

proposed antenna's radiation patterns at frequencies of $2.0 \mathrm{GHz}$, $2.4 \mathrm{GHz}$ and $2.8 \mathrm{GHz}$ have also been investigated. Among these twelve cases, only the effect of L4 at $2.8 \mathrm{GHz}$ is significant, the curves of which are plotted in Fig. 13. It can be seen that the antenna's radiation patterns deflate when L4 is too large or too small. This is because the second resonant frequency shifts away from $2.8 \mathrm{GHz}$, as can be seen in Fig. 9. As a result, the antenna's impedance matching and antenna gain deteriorate. The optimized value for L4 is $32.2 \mathrm{~mm}$.

\section{EXPERIMENTAL RESULTS AND VALIDATION}

To verify the design approach, a prototype of the proposed antenna was fabricated and measured. Fig. 7 gives the detailed dimensions of the manufactured antenna. The reflection coefficient of the antenna was measured by using a R\&S ZNBT 8 24-port vector network analyser while radiation patterns were obtained by employing a far-field antenna measurement system in an EMC screened anechoic chamber.

Fig. 14 shows the simulated and measured reflection coefficient and gain of the proposed antenna. It can be seen that a good agreement is achieved for simulated and measured results. The measured impedance bandwidth shifts slightly to higher frequencies due to the fabrication tolerance. Overall, both the simulated and measured reflection coefficient curves show that the proposed antenna can operate between $1.85 \mathrm{GHz}$ and $2.9 \mathrm{GHz}$ with $\left|\mathrm{S}_{11}\right|<-10 \mathrm{~dB}$. On the other hand, it can also be observed from Fig. 14 that the antenna gain is greater than 2 $\mathrm{dBi}$ across the whole bandwidth.

The electric current distributions of the proposed antenna at its two resonant frequencies, namely $2.0 \mathrm{GHz}$ and $2.75 \mathrm{GHz}$, are depicted in Fig. 15. It can be seen that for the first resonance the current is mainly distributed on the dipole. Though the 
current on the longer side of the loop closer to the dipole is also strong, it barely contributes to the total radiation. At the second resonant frequency, the dipole can be regarded as the couple feeding line of the loop antenna and a folded-dipole-like mode is excited. Therefore, these current distributions further demonstrate our conclusion that the first resonance is caused by the dipole $(\lambda / 2$ mode) while the second resonance is introduced by the loop ( $\lambda$ mode). The phenomenon observed here agrees well with our analysis in Section II.

Fig. 16 shows a great agreement between the simulated and measured radiation patterns. It can be seen that the antenna has an 8-shaped radiation pattern in the XOZ-plane and an omnidirectional pattern in the YOZ-plane over the whole operating frequency band. The measured cross-polarization levels are slightly higher than the simulated results which is mainly caused by the impact of the feed cable. It can also be seen from Fig. 16 that stable radiation patterns are achieved at both E-plane (XOZ-plane) and H-plane (YOZ-plane) across the entire operating frequency band.

\section{CONCLUSION}

A new wideband antenna with stable omnidirectional radiation pattern has been investigated in this communication. The design of the antenna is based on the theory of characteristic modes. After a detailed parametric study, a prototype of the antenna was fabricated and measured. Both the simulated and measured results reveal that the antenna features a wide impedance bandwidth of $44.2 \%\left(\left|\mathrm{~S}_{11}\right|<-10\right)$ from 1.85 to $2.9 \mathrm{GHz}$ and stable radiation patterns are also obtained at both E-plane and H-plane over the operating frequency band. Moreover, the antenna has a simple and planar structure, and it can be built easily by using PCB fabrication technique.

\section{REFERENCES}

[1] C. A. Balanis, Antenna Theory: Analysis and Design, 3rd ed. New York, NY, USA: Wiley-Interscience, 2005.

[2] R. DuHamel and F. Ore, "Logarithmically periodic antenna designs," in IRE Int. Conv. Rec., vol. 6, pp. 139-151, 1958.

[3] J. Dyson, "The characteristics and design of the conical log-spiral antenna," IEEE Trans. Antennas Propag., vol. AP-13, no. 4, pp. 488-499, Apr. 1965.

[4] V. Rumsey, "Frequency independent antennas," in IRE Int. Conv. Rec., vol. 5, pp. 114-118, 1957.

[5] T. W. Chiou and K. L. Wong, "Broad-band dual-polarized single microstrip patch antenna with high isolation and low cross polarization," IEEE Trans. Antenna Propag., vol. 50, no. 3, pp. 399-401, Mar. 2002.

[6] K. F. Lee, K. M. Luk, K. F. Tong, S. M. Shum, T. Huynh, and R. Q. Lee, "Experimental and simulation studies of coaxially fed U-slot rectangular patch antenna," Proc. Inst. Electr. Eng. Microw. Antennas Propag., vol. 144, no. 5, pp. 354-358, Oct. 1997.

[7] C. L. Mak, K. M. Luk, K. F. Lee and Y. L. Chow, "Experimental study of a microstrip patch antenna with an L-shaped probe," IEEE Trans. Antennas Propag., vol. 48, no. 5, pp. 777-783, May. 2000.

[8] J. Y. Sze and K. L. Wong, "Bandwidth enhancement of a microstripline-fed printed wide-slot antenna," IEEE Trans. Antennas Propagat., vol. 49, pp. 1020-1024, Jul. 2001.

[9] S. K. Sharma, L. Shafai and N. Jacob, "Investigation of wideband microstrip slot antenna," IEEE Trans. Antenna and Propagation, vol. 52, no. 3, pp.865-872, Mar. 2004.

[10] S. I. Latif, L. Shafai and S. K. Sharma, "Bandwidth enhancement and size reduction of microstrip slot antenna," IEEE Trans. On Antenna and Propagation, vol. 53, no. 3, pp.994-1003, Mar. 2005.
[11] W. J. Lu and L. Zhu, "Wideband stub-loaded slotline antennas under multi-mode resonance operation," IEEE Trans. Antennas Propag., vol. 63, no. 2, pp. 818-823, Feb. 2015.

[12] E. Safin and D. Manteuffel, "Manipulation of Characteristic Wave Modes by Impedance Loading," IEEE Transactions on Antennas and Propagation, vol. 63, no. 4, pp. 1756-1764, Feb. 2015.

[13] E. Antonino, C. A. Suarez, M. Cabedo, and M. Ferrando, "Wideband antenna for mobile terminals based on the handset PCB resonance," Microw. Opt. Technol. Lett., vol. 48, no. 7, pp. 1408-1411, Jul. 2006.

[14] W. X. Wu and Y. P. Zhang, "Analysis of Ultra-Wideband Printed Planar Quasi-Monopole Antennas Using the Theory of Characteristic Modes," in IEEE Antennas and Propagation Magazine, vol. 52, no. 6, pp. 67-77, Dec. 2010.

[15] M. Bouezzeddine and W. L. Schroeder, "Design of a Wideband, Tunable Four-Port MIMO Antenna System With High Isolation Based on the Theory of Characteristic Modes," in IEEE Transactions on Antennas and Propagation, vol. 64, no. 7, pp. 2679-2688, Jul. 2016.

[16] D. Manteuffel and R. Martens, "Compact Multimode Multielement Antenna for Indoor UWB Massive MIMO," in IEEE Transactions on Antennas and Propagation, vol. 64, no. 7, pp. 2689-2697, Jul. 2016.

[17] K. M. Luk and H. Wong, "A new wideband unidirectional antenna element,” Int. J. Mcrow. Opt. Technol., vol. 1, no. 1, pp. 2098-2101, Jul. 2006.

[18] Q. X. Chu, Y. Luo, "A broadband unidirectional multi-dipole antenna with very stable beamwidth," IEEE Transactions on Antennas and Propagation, vol. 61, no. 5, pp. 2847-2852, May. 2013.

[19] Q. X. Chu, D. L. Wen and Y. Luo, “A Broadband $\pm 45^{\circ}$ Dual-polarized Antenna with Y-shaped Feeding Lines," IEEE Transactions on Antennas and Propagation, vol. 63, no. 2, pp. 483-490, Dec. 2014

[20] Y. H. Cui, R. L. Li and P. Wang, "Novel Dual-Broadband Planar Antenna and Its Array for 2G/3G/LTE Base Stations," IEEE Transactions on Antennas and Propagation, vol. 61, no. 3, pp. 1132-1139, March. 2013.

[21] Z. Bao, Z. Nie, and X. Zong, "A Broadband Dual-Polarization Antenna Element for Wireless Communication Base station," IEEE Asia-Pacific Conference on Antennas and Propagation, pp.144-146, 2012.

[22] R. J. Garbacz, and R. H. Turpin, "A generalized expansion for radiated and scattered fields," IEEE Trans. Antennas Propagat., vol. 19, pp. 348-358, May 1971.

[23] R. F. Harrington, and J. R. Mautz, "Theory of characteristic modes for conducting bodies," IEEE Trans. Antennas Propagat., vol. 19, no. 5, pp. 622-628, Sep. 1971.

[24] R. F. Harrington, and J. R. Mautz, "Computation of characteristic modes for conducting bodies," IEEE Trans. Antennas Propagat., vol. 19, no. 5, pp. 629-639, Sep. 1971.

[25] Y. K. Chen, and C. F. Wang, Characteristic Modes: Theory and Applications in Antenna Engineering. John Wiley \& Sons, 2015.

[26] Altair. CADFEKO Suite7.0 [online]. Available: http://www.feko.info/ product-detail/overview-of-feko.

[27] Ansoft Corp. HFSS [online]. Available: http://www.ansoft.com/products /hf/hfss. 